\title{
NARROW FOCUS ON THE ALBANIAN LANGUAGE ACCORDING TO THE CONCEPT OF LMABRECHT (1994)
}

\author{
Qëndresa Jakupi
}

University of Prishtina

\begin{abstract}
The purpose of this research is to highlight how the focus of the argument or the narrow focus in the Albanian language is structured according to the concept of Lambrecht (1994) for the theory of focus. Lambrecht (1994) suggests that the formal structure of sentences relates to the communicative situations in which sentences are used. According to Lambrecht (1994), the communicative function of the sentence is equal to the focus of the sentence, whereas the focus of a sentence always contains the new information that is said in that sentence.Based on the communicative function it divides the focus into three types; sentence focus, predicate focus, and narrow focus. Narrow focus, which is also the object of our research, is defined by Lambrecht as a domain of focus that is limited to a single constituent (subject, predicate, adjunct, object etc.).This type of focus has been identified within our corpus, which consists of commentary on the Albania-Serbia match (October 14, 2014) and the newspaper Koha, in which case the way is analyzed how this focus is codified within the declarative sentences detached from the corpus of the work.
\end{abstract}

Keywords: narrow focus, Lambrecht concept (1994), declarative sentence, Koha newspaper, match commentary.

\section{Introduction}

Lambrecht (1994) suggests that formal sentence structure relates to communicative situations in which sentences are used. He states that this relationship is governed by the principles and rules of grammar, in a component called the information structure (1994: 334). Therefore, depending on the communicative function of the sentences, the syntactic structures also change (the same is argued by Givon 1983, 1988; Hawkins 1991). According to Lambrecht (1994), the functions of communication and the sentence are equal to the focus of the sentence. Lambrecht (ibid. 14) conceives of focus structure as something that conventionally connects sentence form with focus construct. The syntactic field in a sentence which expresses the focus component of a pragmatically structured sentence in the field of focus (ibid. 241). Among the topics of the taxonomy of focus structures that were developed is the type of focus of the sentence, i.e., type in which the scope of the new information extends over the entire proposal, including the subject. To extract the focus of the sentence, a question is asked through which the answer does not give the presumption. When asked what happened, for example, by someone who has no prior knowledge of the event, a speaker cannot give answer, starting the sentence with a specific topic. Thus, no pragmatic presupposition is evoked, so the whole answer is mostly new information and, therefore, is the focus of the sentence. Schematic presentation:

Sentence: My car broken

Presupposition: ------

Assertion: "speaker's car broke down"

Focus: "speaker's car broke down"

Focus domain: $S$ 
Since the assertion extends over the whole proposition, here the assertion and the focus coincide. Consequently, in these structures, the field of focus is the whole sentence (ibid.233).

So, the communicative function of such focus structures in the sentence is either to introduce a new reference to the discourse or (as in this case) to present an event involving a new reference to the discourse. For example:

My car is broken.

The italic shaded part is the focus. Whereas, the new reference in the discourse would be not my car, but the woman's car. Apart from the fact that the focus of the sentence is indicated through a new discourse reference, according to Lambrecht, the focus is also marked by the place of the main accent of the sentence, which is characterized by the pitch of the tone.The emphasis of the sentence serves as a marker of focus, i.e. as the formal indicator of the sentence structure of the sentence. He also gives examples even when the emphasis of the sentence falls only on the topic or keyword, the focus area as the domain of new information extends throughout the sentence. The emphasis of the sentence serves as a marker of focus, i.e. as the formal indicator of the sentence structure of the sentence. He also gives examples even when the emphasis of the sentence falls only on the topic or keyword, the focus area as the domain of new information extends throughout the sentence: predicate, subject, adjunct, accusative. So here, we are talking about the other two types of focus, predicate focus and narrow focus.

Predicate focus is the type of universally unmarked focus structure. Pragmatic presupposition in this kind involves knowing a certain topic, and the assertion expresses a comment about the topic. The predicate focus, in other words, is a topic-comment structure where the comment, or predicate, is in focus. For example, person S1 may know that something happened to person S2's car. When person S1 asks S2 about the car, in response to S2, the car is considered a presupposed element, while what happened to the car as new information, i.e., focus. The information structure is schematically presented as follows:

Sentence: My car broke DOWN.

Presupposition: speaker's car is a topic for $x$ comment.

Assertion: " $x=$ broke down"”

Focus: "broke down"”

Focus domain: VP

The question is about the $\mathrm{S} 2$ machine, which forms the presupposition for the answer: the speaker car is the topic for comment $x$. The assertion is that $x=$ was broken. Therefore, the assertion creates a relationship between the topic reference and the state of the problem referred to by the predicate was broken, which is the focus. The field of focus in this type of structure is the verbal component and, when the verb is transitive, it also includes the direct object.

The second type of focus structure is a narrow focus. Lambrecht also refers to it as the focus of the argument (1994: 228). In the narrow or focus structure, the focus domain is limited to a single component (subject, object, adjunct, etc.). For example:

S1: I heard your engine broken down.

S2: My CAR broke down. 
From the example above it is understood that the focus of the argument is not the component itself, but establishing a relationship between the reference and the assumed proposition "something the speaker possesses that is broken", in creating new information that it is the speaker's car that broke. The relationship between 'car' and the verb 'was broken' is asserted, but the assertion is not a simple identification of $\mathrm{X}$ with the speaker's car, but also the correction of a misconception (Van Valin and LaPolla 1997: 209).

In the given sentence something possessed by the speaker is broken and this is part of the pragmatic presupposition, while the assertion is that the speaker's car was broken, and the focus is the car, and the focus area is the whole Domain. The field of focus is limited to a single component. Since in this particular example the open proposition $\mathrm{x}$ was broken it is active and not my car reference, making the activation status of the two parts of the statement in this example. Schematic presentation:

Sentence: My CAR broke down.

Presupposition: "speaker's $x$ broke down"

Assertion: $x=$ 'car'

Focus: "car"

Focus domain: NP

From the point of view of the current communicative situation in which it is pronounced, the schematic representation in the example above is considerably specified. In the given situation, the common point between the speaker and the addressee is not simply that something belonging to the speakeris broken, but that the addressee thinks that this something that is broken is the speaker's engine. The assertion made by uttering this sentence is not merely the identification of $\mathrm{X}$ with the speaker's car, but also the correction of a misconception on the part of the recipient (Lambrecht 1994: 229).

Lambrecht (1986) also distinguishes unmarked narrow focus from narrow marked focus. The main difference is related to the location of the component focused in the sentence, if the component as the narrow focus is in the final position of the sentence, it is unmarked, while if the component as the narrow focus is shifted to the left and not at the end of the sentence, then it is marked. For example:

1. Bekim Balaj tries there marked

2. There tries BekimBalaj unmarked

Thus, a narrow focus on contrast is a case of unbranded narrow focus, whereas a narrow focus on a subject is a case of narrow marked focus.

\subsection{Methods and Methodology}

To analyze the way a narrow focus is codified in the Albanian language, some declarative sentences have been identified which in their structure enclose the narrow focus. These sentences were collected from the corpus which constitutes this research, ie from the commentary of the Albania-Serbia match (October 14, 2014) and the newspaper Koha. While the methodology used to analyze the narrow focus within these sentences is based on the methodology and criteria used by Lambrecht in his book Information structure and sentence form. Topic, focus, and the representation of mental referents in discourse (1994). 


\section{Analysis}

\section{Examples of narrow focus}

Example 1: // Must be a corner kick //

Context of the sentence: What should this be?

Sentence: Must be a corner kick

Presupposition: "the ball has deviated"

Assertion: corner kick

Focus: corner kick

Focus Domain: DP

The example above establishes a relationship between the reference and the supposed "corner kick" proposition, as the commentator does not see well if the deflected ball has gone out of the sideline or bottom line. Therefore, the DP lateralization creates new information in this sentence. In this structure the pragmatic presupposition is that the ball is deflected, the assertion is that it should be a corner kick, the focus is a 'corner kick' and the focus area is the entire DP. The field of focus is limited to a single component.

Example 2: // There tries Bekim Balaj//

Sentence context: Who started the quick action?

Sentence: There tries Bekim Balaj

Presupposition: Someone started a quick action

Assertion: Bekim Bala

Focus: Bekim Balaj

Focus Domain: DP

From the example above, a relationship is established between the reference and the supposed proposal "Bekim Balaj", since it can be seen that one of the players has started a quick action. Therefore, DP Bekim Balaj creates new information in this sentence. Because the one who started a quick action towards the Serbian gate is Bekim Balaj.

Example 3: // There are corner kicks for Serbs //

Context of the sentence: For whom is the corner kick?

Sentence: There are corner kicks for Serbs

Presupposition: We have a corner kick

Assertion: from Serbs

Focus: from Serbs 


\section{Focus Domain: PP}

In this structure, the pragmatic assumption is that we have corner kicks, while the assertion is that this kick belongs to the Serbs, the focus is "by the Serbs" and the focus area is the whole PP. The field of focus is limited to a single component.

Example 4: Prishtina squares are illuminated

Context of the sentence: What was enlightened in Prishtina?

Sentence: Prishtina squares are illuminated

Presupposition: Squares

Assertion: Squares

Focus Domain: DP

In this example, a relationship is established between the reference and the supposed "squares" proposal, as the listener does not know for sure what was illuminated in Prishtina. In this structure there is a pragmatic presupposition that something has been illuminated in Prishtina, the assertion is that the squares have been illuminated, the focus is on the "squares" and the focus area is the entire DP. Therefore, the field of focus is narrowed to a single component.

\section{Conclusion}

Based on the criteria of Lambrecht (1994) that have been made to identify and analyze how the narrow focus is codified in a language, which has been applied in this research, in which case it has been noticed that in the Albanian language there is Lambrecht's concept of narrow focus and whose field is limited to a single component and that component can function in sentences, such as subject, adjunct, object etc. Besides the field of focus can be limited within a DP, PP, or even within a nominal group, so it turns out that the focus is not always coded through a verb or a VP. Also, in order to determine the field of focus in the Albanian language, extra-linguistic factors are important, such as the connection between the reference and the supposed proposal, as well as the pragmatic assertion and presupposition of what is said. 


\section{Bibliography}

1. Givon, Talmy (1983). Topic Continuity in Discourse: A Quantitative Cross-Language Study. Amsterdam: John Benjamins.

2. Givon, Talmy. (1988): The pragmatics of word order: Predictability, importance and attention. In: Studies in Syntactic Typology, ed. by M. Hammond, E. A. Moravcsik \& J. Wirth. Amsterdam: John Benjamins, $243-284$.

3. Halliday, Michael (1985 [2014]). Introduction to Functional Grammar. University of Birmingham. United Kingdom

4. Hawkins, John A. (1991). Syntactic weight versus information structure in word order variation. Linguistische Berichte. Special issue on Information Structure and Grammar.

5. Huddleston, R. Pullum, G. K. (2005). A studnet's introduction to English Grammar. Cambridge University Press.

6. Lambrecht Kund (1994). Information structure and sentence form. Topic, focus, and the representation of mental referents in discourse. Cambridge: Cambridge University Press.

7. Lambrecht, Kund (1986). Topic, Focus and the Grammar of Spoken French. PhD dissertation, University of California Berkeley.

8. Rugova Bardh (2006 dhe 2007). Gjuha e sportit. Filologji 14/15, Universiteti i Prishtinës, Prishtinë, pp 141 - 156.

9. Rugova Bardh (2009). Gjuha e gazetave. Koha. Prishtinë.

10. Van Valin, Robert D. and LaPolla, Randy J. (1997). Syntax: Structure, Meaning, and Function. Cambridge: Cambridge University Press. 